\title{
Generalized logistic distribution and its regression model
}

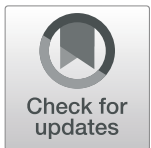

\author{
Mohammad A. Aljarrah1*, Felix Famoye ${ }^{2}$ and Carl Lee $^{2}$
}

\author{
* Correspondence: aljarrah@ymail. \\ com \\ ${ }^{1}$ Department of Mathematics, Tafila \\ Technical University, Tafila 66110, \\ Jordan \\ Full list of author information is \\ available at the end of the article
}

\begin{abstract}
A new generalized asymmetric logistic distribution is defined. In some cases, existing three parameter distributions provide poor fit to heavy tailed data sets. The proposed new distribution consists of only three parameters and is shown to fit a much wider range of heavy left and right tailed data when compared with various existing distributions. The new generalized distribution has logistic, maximum and minimum Gumbel distributions as sub-models. Some properties of the new distribution including mode, skewness, kurtosis, hazard function, and moments are studied. We propose the method of maximum likelihood to estimate the parameters and assess the finite sample size performance of the method. A generalized logistic regression model, based on the new distribution, is presented. Logistic-log-logistic regression, Weibull-extreme value regression and log-Fréchet regression are special cases of the generalized logistic regression model. The model is applied to fit failure time of a new insulation technique and the survival of a heart transplant study.
\end{abstract}

Keywords: Beta-family, Symmetric distribution, Hazard function, Moments, Censored data

2010 Mathematics subject classification: 62E15, 62F10, 62 J12, 62P10

\section{Introduction}

The use of logistic distribution in various disciplines can be found in (Johnson et al. 1995) and the references therein. The logistic distribution has the cumulative distribution function $(\mathrm{CDF})$ defined as

$$
F(x)=\left(1+\exp \left(-\frac{x-\mu}{\sigma}\right)\right)^{-1},-\infty<x, \mu<\infty, \sigma>0
$$

Note that the logistic distribution is the limiting distribution of the average of largest and smallest values of random samples of size $n$ from a symmetric distribution of exponential type (Gumbel 1958).

The CDF of the standard logistic distribution is $F(y)=\left(1+e^{-y}\right)^{-1},-\infty<y<\infty$. The standard logistic density function with kurtosis 4.2 is symmetric about zero, and is more peaked and has heavier tails than the normal density function. These properties make logistic distribution a popular choice for fitting symmetric non-normal data.

(c) The Author(s). 2020 Open Access This article is licensed under a Creative Commons Attribution 4.0 International License, which permits use, sharing, adaptation, distribution and reproduction in any medium or format, as long as you give appropriate credit to the original author(s) and the source, provide a link to the Creative Commons licence, and indicate if changes were made. The images or other third party material in this article are included in the article's Creative Commons licence, unless indicated otherwise in a credit line to the material. If material is not included in the article's Creative Commons licence and your intended use is not permitted by statutory regulation or exceeds the permitted use, you will need to obtain permission directly from the copyright holder. To view a copy of this licence, visit http://creativecommons.org/licenses/by/4.0/. 
The first type of extreme value distribution is commonly known as the Gumbel-type distribution due to Gumbel (1958), who made several significant contributions to the extreme value analysis and practical applications of extreme value statistics in distributions of human lifetimes, radioactive emissions, and flood analysis (see, e.g., Johnson et al. 1995). Gumbel used the distribution to model the maximum and minimum values of samples from various distributions. The CDFs of maximum and minimum Gumbel distributions are defined, respectively, as

$$
\begin{aligned}
& F_{G u-\max }(x ; \mu, \sigma)=\exp \left\{-\exp \left(-\frac{x-\mu}{\sigma}\right)\right\},-\infty<x<\infty,-\infty<\mu<\infty, \sigma>0, \\
& F_{G u-\min }(x ; \mu, \sigma)=1-\exp \left\{-\exp \left(\frac{x-\mu}{\sigma}\right)\right\},-\infty<x<\infty,-\infty<\mu<\infty, \sigma>0,
\end{aligned}
$$

where $\mu$ and $\sigma$ are location and scale parameters, respectively. Gumbel distribution is good to fit skewed data while logistic distribution is for symmetric data. It is interesting to note that there is a relation between these two distributions. If $X \sim \operatorname{Gumbel}\left(\mu_{X}, \sigma\right)$ and $Y \sim \operatorname{Gumbel}\left(\mu_{Y}, \sigma\right)$, then, $(X-Y) \sim \operatorname{logistic}\left(\mu_{X}-\mu_{Y}, \sigma\right)$.

In order to improve the goodness of fit of the logistic and Gumbel distributions, many generalizations of these distributions have been studied in the literature. For example, Prentice (1976) proposed logistic type IV to model binomial regression data. Stukel (1988) proposed logistic regression model. Balakrishnan and Leung (1988) proposed three types of generalized logistic distribution. Johnson et al. (1995) summarized several generalizations of the logistic distribution. Wahed and Ali (2001) proposed the skew logistic distribution (SLD). An extension of SLD was presented and studied by Nadarajah (2009) by introducing a scale parameter. Gupta and Kundu (2010) defined two generalizations of logistic distribution, namely the skew logistic using the skew normal method proposed by Azzalini (1985) and defined the Type-II logistic distribution as a member of the proportional reversed hazard family with the baseline distribution as the logistic distribution. The $T$ - $X$ framework proposed by Alzaatreh et al. (2013), which was further expanded by Aljarrah et al. (2014) are two general methods that have been applied to derive various generalization of distributions, including logistic distribution. Recently, Ghosh and Alzaatreh (2018) defined the exponentiatedexponential logistic (EEL) distribution as a generalization of the logistic distribution and various properties were studied by the authors.

Similar to the logistic distribution, several generalizations of the Gumbel distribution have appeared in the literature. For a review of generalizations of the Gumbel extreme value distribution, one may refer to Pinheiro and Ferrari (2016).

There is already a long list of literatures for generalized logistic and Gumbel distributions. Why are we developing yet another family of generalized logistic distributions? As pointed out by Johnson et al. (1994, p. 15) "For most practical purposes, it is sufficient to use four parameters. There is no doubt that at least three parameters are needed; for some purposes this is enough." The main motivation is to develop highly flexible three-parameter distributions that can fit wide range of right and left skewed data. The method proposed here has several advantages that are not available among the existing generalizations: 
(a) The method proposed is not to develop a single generalized logistic distribution, it can be applied to generate different families of generalized logistic distributions. A generalized normal distribution using similar technique was studied in Aljarrah et al. (2019), which was shown to be a much more flexible distribution than the skewed normal proposed by Azzalini (1985) and its generalizations.

(b) A member of the family of the generalized logistic distributions, the exponentiallogistic $\{$ Generalized Weibull\} distribution (E-L $\{G W\}$ ) is defined and studied in detail in this article. This distribution has three parameters: location, scale and a shape parameter. As shown in the article, the E-L $\{G W\}$ distribution is a generalization of both logistic and Gumbel distributions.

(c) The E-L $\{G W\}$ is shown to be more flexible than existing generalizations of logistic and Gumbel distributions in two ways: (i) It fits very well left and right skewed data. Existing generalized logistic or Gumbel distributions can fit heavy rightskewed data, but not able to fit heavy left-skewed data. (ii) It fits very well data with a wider range of skewness and kurtosis when compared with existing generalizations such as skew logistic (Gupta and Kundu 2010), beta-logistic distribution (Nassar and Elmasry 2012), generalized logistic distribution (Ghosh and Alzaatreh 2018), generalized Gumbel (Cooray 2010), as well as skew normal (Azzalini 1985) and its five-parameter generalized distribution (Choudhury and Abdul 2011).

(d) The generalized regression model derived by assuming the response follows E-L $\{G W\}$ distribution is a very flexible model that takes logistic-log-logistic regression, Weibull-extreme value regression and log-Fréchet regression as special cases.

In Section 2, we define the E-L $\{G W\}$ distribution. Some properties of the E-L $\{G W\}$ distribution including the shapes of the probability density function (PDF) and hazard function, and quantile function are studied. An expression for the moment, properties of the hazard function, and the relationship between the mean, variance, skewness, kurtosis and the shape parameter are investigated in Section 3. In Section 4, the method of maximum likelihood is presented for estimating the parameters of the distribution, and a simulation study is performed to assess the small sample performance of the method. In Section 5, a generalized logistic regression model based on E-L $\{G W\}$ distribution is developed. In Section 6, applications to several real data sets are given to demonstrate the flexibility and usefulness of the new distribution and its regression model. Summary and conclusions are given in Section 7.

\section{The exponential-logistic \{generalized Weibull\} (E-L \{GW\}) distribution}

Let the random variable $R$ be a standard logistic distribution. Using a shape parameter $\xi>0$, location parameter $-\infty<\mu<\infty$, scale reflection parameter $\sigma \neq 0$, and following the technique that Aljarrah et al. (2019) used to define the combined exponentialnormal $\{G W\}$ distribution, we define the combined $E-R\{G W\}$ family as

$$
F_{X}(x)=0.5+\operatorname{sgn}(\sigma)\left(0.5-\exp \left\{-\left(\left(\bar{F}_{R}\left(\frac{x-\mu}{\sigma}\right)\right)^{-\xi}-1\right) / \xi\right\}\right)
$$

where $\operatorname{sgn}(\sigma)$ is the sign of the parameter $\sigma$. Note that the CDF defined in (4) reduces to $F_{R}\left(\frac{x-\mu}{|\sigma|}\right)$ distribution as $\xi \rightarrow 0$. The corresponding PDF to (4) is given by 


$$
f_{X}(x)=\frac{f_{R}\left(\frac{x-\mu}{\sigma}\right)}{|\sigma|\left(\bar{F}_{R}\left(\frac{x-\mu}{\sigma}\right)\right)^{\xi+1}} \exp \left(-\frac{\left(\bar{F}_{R}\left(\frac{x-\mu}{\sigma}\right)\right)^{-\xi}-1}{\xi}\right) .
$$

The E-L $\{G W\}$ distribution can be defined from Eq. (4) by letting $R$ be the logistic random variable as follows:

Definition (E-L $\{G W\}$ distribution): The CDF and PDF of the E-L $\{G W\}$ distribution are defined, respectively, as

$$
F_{X}(x)=0.5+\operatorname{sgn}(\sigma)\left(0.5-\exp \left\{\left(1-\left(1+\exp \left(\frac{x-\mu}{\sigma}\right)\right)^{\xi}\right) / \xi\right\}\right)
$$

and

$$
\begin{aligned}
f_{X}(x)= & \frac{1}{|\sigma|} \exp \left(\frac{x-\mu}{\sigma}\right)\left(1+\exp \left(\frac{x-\mu}{\sigma}\right)\right)^{\xi-1} \exp \left\{\left(1-\left(1+\exp \left(\frac{x-\mu}{\sigma}\right)\right)^{\xi}\right) / \xi\right\}, \\
& -\infty<x, \mu<\infty, \sigma \neq 0, \xi>0 .
\end{aligned}
$$

Note the E-L $\{G W\}$ is derived as a generalization of the symmetric logistic distribution for fitting highly skewed data. This provides a good comparison of performance when comparing with various existing three-parameter distributions. The following Corollary presents some special sub-models.

Corollary 1: The PDF of $\mathrm{E}-\mathrm{L}\{\mathrm{GW}\}(\mu, \sigma, \xi)$ in (7) reduces to the following submodels:

a) When $\xi \rightarrow 0$, the PDF in (7) reduces to a logistic distribution in (1).

b) When $\xi=1$ and $\sigma<0$, the PDF in (7) reduces to the PDF of maximum Gumbel distribution in (2) with location and scale parameters $\mu$ and $|\sigma|$, respectively.

c) When $\xi=1$ and $\sigma>0$, the PDF in (7) reduces to the PDF of minimum Gumbel distribution in (3) with location and scale parameters $\mu$ and $\sigma$, respectively.

Proof: a) $\lim _{\xi \rightarrow 0} f_{X}(x)=\frac{1}{|\sigma|} \exp \left(\frac{x-\mu}{|\sigma|}\right) /\left(1+\exp \left(\frac{x-\mu}{|\sigma|}\right)\right)^{2}$, that is $X \sim$ Logistic $(\mu,|\sigma|)$. The cases (b) and (c) are obtained directly by substituting $\xi=1$ in (7).

Quantile functions are useful for generating pseudo-random numbers from a probability distribution. Proposition 1 gives the quantile function for the E-L $\{G W\}$ distribution.

Proposition 1: The quantile function for the E-L $\{G W\}$ distribution is given by

$$
Q_{X}(u)=\mu+\sigma \log \left(\left\{1-\xi \log \left(\frac{1}{2}-\operatorname{sgn}(\sigma)\left(u-\frac{1}{2}\right)\right)\right\}^{1 / \xi}-1\right), u \in(0,1) .
$$

Proof: By setting $F_{X}\left(Q_{X}(u)\right)=u$ in Eq. (6) and solving for $Q_{X}(u)$ in terms of $u$, the quantile function in (8) is obtained. $\square$ 


\section{Proposition 2:}

a) If $T$ is a standard exponential random variable, then $X=\mu+\sigma \log \left((1+\xi T)^{1 / \xi}-1\right)$ follows the E-L $\{\mathrm{GW}\}(\mu, \sigma, \xi)$ distribution in Eq. (6).

b) If $X \sim \mathrm{E}-\mathrm{L}\{\mathrm{GW}\}(\mu, \sigma, \xi)$, then $(2 \mu-X) \sim \mathrm{E}-\mathrm{L}\{\mathrm{GW}\}(\mu,-\sigma, \xi)$.

Proof: Using the CDF method, the results in (a) and (b) follow.

The hazard rate function (HRF) of the E-L $\{G W\}$ distribution is obtained after using the CDF in (6) and PDF in (7), and it is given by

$$
h(x)= \begin{cases}\frac{1}{\sigma} \exp \left(\frac{x-\mu}{\sigma}\right)\left\{\exp \left(\frac{x-\mu}{\sigma}\right)+1\right\}^{\xi-1}, & \sigma>0, \\ \frac{\exp \left(\frac{x-\mu}{\sigma}\right)\left\{\exp \left(\frac{x-\mu}{\sigma}\right)+1\right\}^{\xi-1}}{|\sigma|\left\{\exp \left(\frac{1}{\xi}\left[\left\{\exp \left(\frac{x-\mu}{\sigma}\right)+1\right\}^{\xi}-1\right]\right)-1\right\}}, & \sigma<0 .\end{cases}
$$

Figures 1 and 2 show the plots of PDF and HRF for E-L \{GW\} distribution. The PDF can be positively or negatively skewed, while the HRF shows increasing with J shape, increasing with $\mathrm{S}$ shape, and increasing-decreasing shapes. The graphs in Fig. 1 indicate that the distribution tends to be symmetric as $\xi \rightarrow 0$, skewed to the left when $\sigma>0$, and skewed to the right when $\sigma<0$. When the sign of parameter $\sigma$ is changed, the curve of the PDF is reflected about the line $x=0$. Also as $\xi$ increases, the mode decreases when $\sigma>0$, and as $\xi$ increases, the mode increases when $\sigma<0$. The graphs in Fig. 2 show the hazard function in (9) is increasing when $\sigma>0$. When $\sigma<0$, the hazard function increases or first constant, increases and then decreases.

\section{Properties of exponential-logistic \{generalized Weibull\} distribution}

In this section, some properties of the E-L $\{G W\}$ distribution are studied. These properties include, mode, shape property of the HRF, moments and moment generating function.
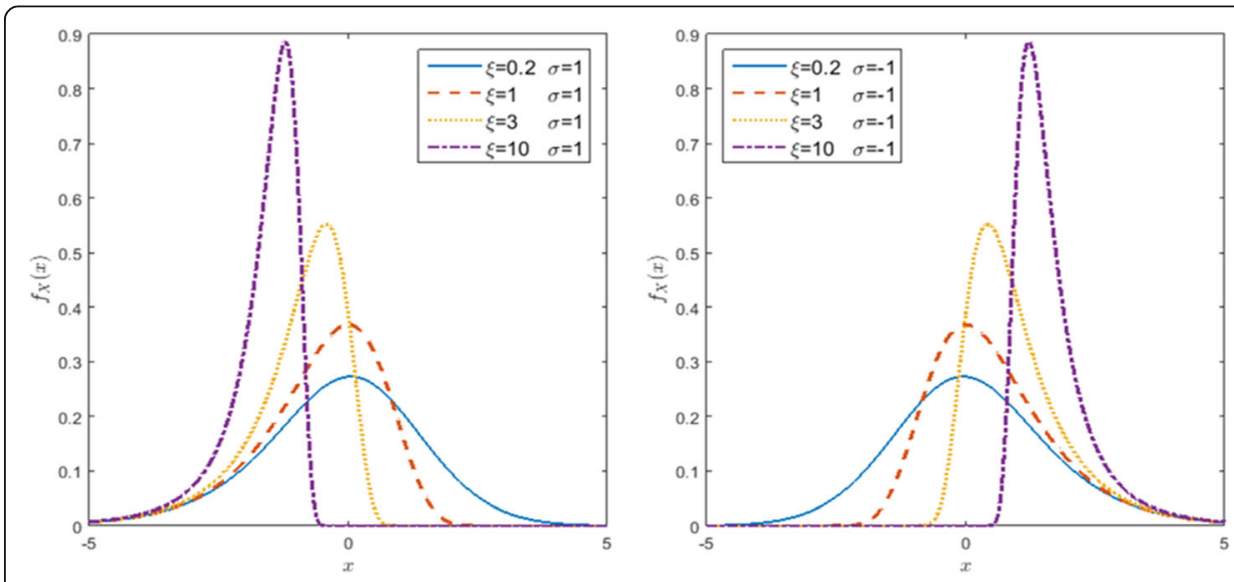

Fig. 1 Plots of PDF of $E-L\{G W\}$ distribution with $\mu=0$ 

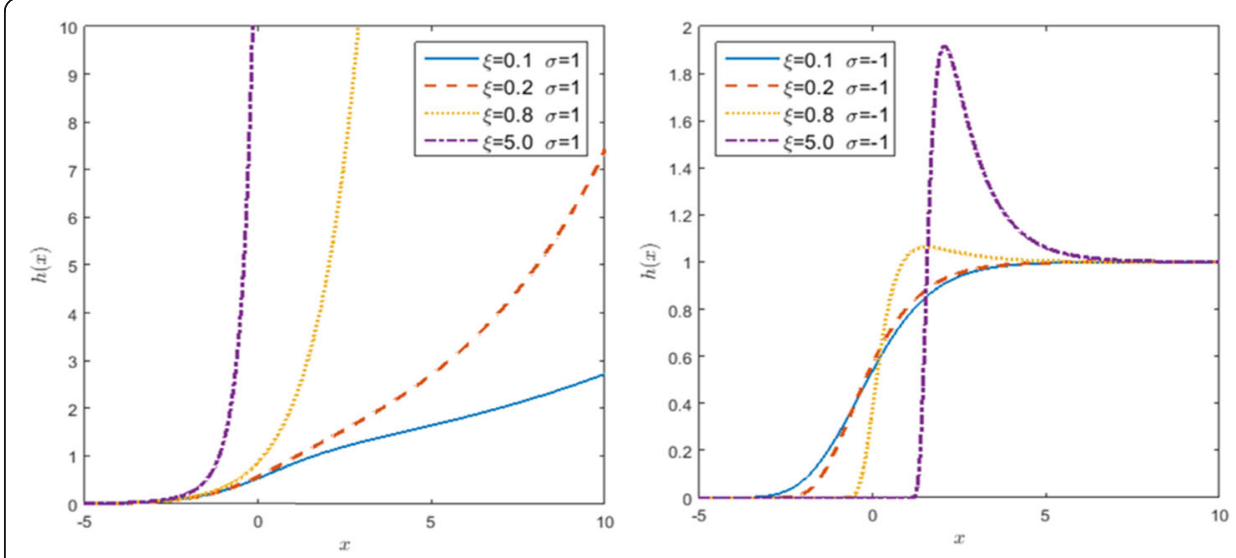

Fig. 2 Plots of HRF of $E-L\{G W\}$ distribution with $\mu=0$

\section{Mode:}

Theorem 1: The E-L $\{G W\}$ distribution is unimodal. The mode is at the point $x_{*}=\mu$ whenever $\xi=\{0,1\}$. Otherwise the mode is at the point $x_{*}=\mu+\sigma \log \left(u_{*}\right)$, where $u_{*}$ satisfies the equation

$$
\xi u+1=u(u+1)^{\xi}, u>0 .
$$

Proof: See Appendix.

Corollary 2: The HRF is increasing whenever $\sigma>0$, and asymptotic to the line $y=1$ / $|\sigma|$ as $x \rightarrow \infty$ whenever $\sigma<0$.

Proof: See Appendix.

It is noteworthy to mention that the graphs in Fig. 2 are consistent with the above results and the asymptotic feature of the curves in Corollary 2.

Moments: The moments are valuable for describing and identifying distribution properties such as the center, variance, skewness and kurtosis. In order to derive the moments of E-L $\{G W\}$, we first provide a series expansion of PDF of E-R $\{G W\}$ in Eq. (5), by applying the exponential series, as follows.

$$
f_{X}(x)=\frac{f_{R}\left(\frac{x-\mu}{\sigma}\right) \exp (1 / \xi)}{|\sigma|} \sum_{i=0}^{\infty} \frac{(-1)^{i}\left(\bar{F}_{R}\left(\frac{x-\mu}{\sigma}\right)\right)^{-(\xi i+\xi+1)}}{i ! \xi^{i}} .
$$

By applying negative binomial series expansion $(1-x)^{-r}=\sum_{j=0}^{\infty} \frac{\Gamma(r+j)}{\Gamma(j+1) \Gamma(r)} x^{j},|x|<1$ on $\left(\bar{F}_{R}((x-\mu) / \sigma)\right)^{-(\xi i+\xi+1)}$, we get

$$
\begin{gathered}
f_{X}(x)=\frac{f_{R}\left(\frac{x-\mu}{\sigma}\right) \exp (1 / \xi)}{|\sigma|} \sum_{i=0}^{\infty} \sum_{j=0}^{\infty} \frac{(-1)^{i} \Gamma(\xi i+\xi+j+1)}{\xi^{i} i ! \Gamma(j+1) \Gamma(\xi i+\xi+1)}\left(F_{R}\left(\frac{x-\mu}{\sigma}\right)\right)^{j} \text {, which can be written as } \\
\left.f_{X}(x)=\sum_{i=0}^{\infty} \sum_{j=0}^{\infty} \frac{\omega_{i, j}}{|\sigma|} k_{(j+1)}((x-\mu) / \sigma)\right) .
\end{gathered}
$$

where 


$$
\omega_{i, j}=\frac{(-1)^{i} \exp (1 / \xi) \Gamma(\xi i+\xi+j+1)}{\xi^{i} i ! \Gamma(j+2) \Gamma(\xi i+\xi+1)},
$$

and $k_{(j+1)}(x)=(j+1) f_{R}(x)\left(F_{R}(x)\right)^{j}$ denotes the PDF of exponentiated $R$ random variable with power parameter $j+1$.

Theorem 2: The $n^{\text {th }}$ absolute moment of the $E-L\{G W\}$ distribution exists for any $\mu$, $\sigma \neq 0, \xi>0$ and satisfies the inequality

$$
E\left(|X|^{n}\right) \leq e^{-1}(1+\xi)^{1+1 / \xi} \sum_{i=0}^{n}\left(\begin{array}{l}
n \\
i
\end{array}\right)|\mu|^{n-i}|\sigma|^{i} E\left(|L|^{i}\right),
$$

where $L$ is a standard logistic random variable.

Proof: See Appendix.

Moments of E-L $\{G W\}$ as a series expression is given in the following theorem.

Theorem 3: The $r^{\text {th }}$ moment, $E\left(X^{r}\right)$, of the $E-L\{G W\}$ distribution is given by

$$
E\left(X^{r}\right)=\sum_{n=0}^{r} \sum_{i=0}^{\infty} \sum_{j=0}^{\infty}\left(\begin{array}{l}
r \\
n
\end{array}\right) \mu^{r-n} \sigma^{n} \omega_{i, j} E\left(L_{j+1}^{n}\right)
$$

where $\omega_{i, j}$ is defined in (12) and $E\left(L_{i+1}^{n}\right)$ is the $n^{\text {th }}$ moment of the exponentiated logistic distribution with power parameter $j+1$ and given by Ali et al. (2007) as

$$
E\left(L_{j+1}^{n}\right)=(j+1) n !\left(\sum_{k=0}^{\infty} \frac{(-j-2) P_{k}}{k !(k+1)^{n+1}}+(-1)^{n} \sum_{k=0}^{\infty} \frac{(-j-2) P_{k}}{k !(k+j+1)^{n+1}}\right) .
$$

Proof: See Appendix.

Proposition 3: Suppose $X$ has the PDF in (6), then the moment generating function (MGF) of $X$ is given by

$$
M_{X}(t)=e^{\mu t+1 / \xi} \sum_{i=0}^{\infty} \frac{\Gamma(\sigma t+1)(-1)^{i}}{\Gamma(\sigma t-i+1) \Gamma(i+1)} \xi^{(\sigma t-i) / \xi} \Gamma((\sigma t-i) / \xi+1,1 / \xi),
$$

where
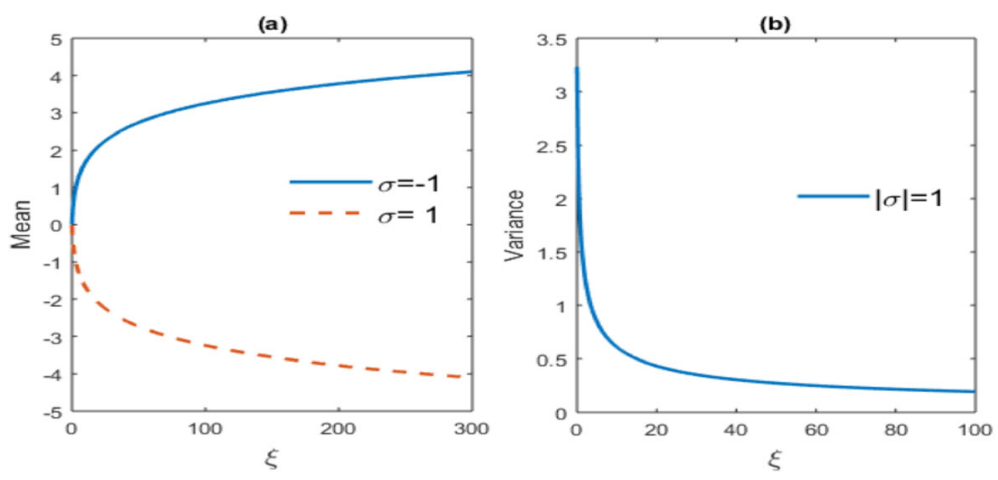

Fig. 3 Graphs of mean, and variance for $E-L\{G W\}$ distribution for $\mu=0, \sigma=\{1,-1\}$, and various values of $\xi$ 

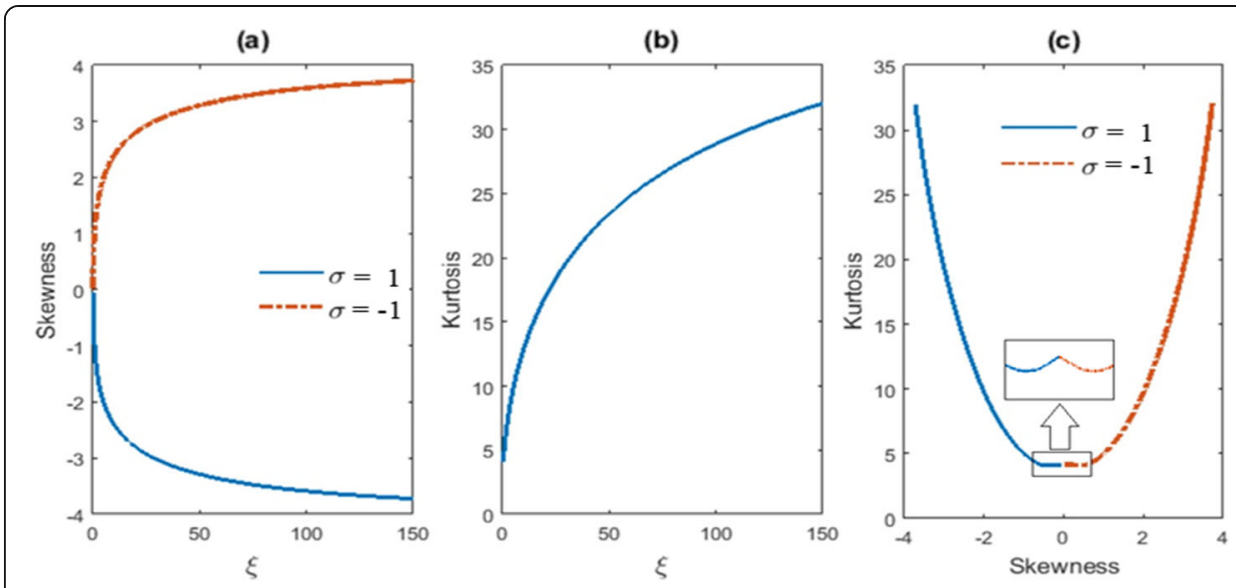

Fig. 4 The skewness and kurtosis for $E-L\{G W\}$ distribution for $\mu=0$ and $\sigma=\{1,-1\}$ for various values of $\xi$

$$
\begin{cases}t \in(-\infty, 1 /|\sigma|), & \xi \geq 1, \sigma<0 \\ t \in(-1 / \sigma, \infty), & \xi \geq 1, \sigma>0 \\ t \in(-1 /|\sigma|, 1 /|\sigma|), & \xi<1, \sigma \neq 0\end{cases}
$$

Proof: See Appendix.

In Fig. 3, the mean and variance of E-L $\{\mathrm{GW}\}$ distribution are plotted in terms of the parameter $\xi$ for $\mu=0$ and $\sigma=\{1,-1\}$. Figure 3(a) shows that when $\sigma>0$, the mean decreases as $\xi$ increases, When $\sigma<0$, the mean increases as $\xi$ increases. Also, Fig. 3(b) shows that the variance decreases as $\xi$ increases.

In Fig. 4, we plot the skewness and kurtosis of E-L $\{\mathrm{GW}\}$ distribution in terms of the parameters $\xi$ when $\mu=0$ and $\sigma=\{1,-1\}$. Figure 4(a) shows that when $\sigma>0$, the skewness decreases as $\xi$ increases and the E-L $\{\mathrm{GW}\}$ distribution is left skewed, and when $\sigma<0$, the skewness increases as $\xi$ increases and the E-L \{GW\} distribution is right skewed. The distribution is symmetric as $\xi \rightarrow 0$. We note that the degree of skewness of the E-L $\{\mathrm{GW}\}$ distribution is measured by $\xi$, and the parameter $\sigma$ plays two roles: characterizing the scale property and determining left skewed $(\sigma>0)$ or right skewed $(\sigma<0)$. Figure 4(b) shows the kurtosis increases as $\xi$ increases, and it is not affected by $\sigma$.

Table 1 A comparison of skewness and kurtosis of some generalized logistic and Gumbel distributions

\begin{tabular}{lll}
\hline Distribution & \multicolumn{1}{l}{ Skewness } & Kurtosis \\
\hline SN & -0.995 to 0.995 & 3.000 to 3.869 \\
ESGN & -0.995 to 0.995 & 3.000 to 7.238 \\
GN & -3.000 to 3.000 & 3.000 to 20.000 \\
BGL & -2.000 to 2.000 & 3.000 to 6.000 \\
PRHL & -2.000 to 1.140 & 1.138 to 6.000 \\
GG & -1.500 to 1.500 & 4.245 to 6.200 \\
EEL & -1.080 to 1.889 & 2.393 to 8.553 \\
E-L \{GW\} & -4.000 to 4.000 & 3.956 to 35.000 \\
\hline
\end{tabular}


The flexibility of the E-L $\{\mathrm{GW}\}$ is compared with skew normal (SN) (Azzalini 1985), extended skew generalized normal (ESGN) (Choudhury and Abdul 2011), generalized normal (GN) (Aljarrah et al. 2019), beta-generalized logistic (BGL) (Nassar and Elmasry 2012), proportional reversed hazard logistic (PRHL) (Gupta and Kundu 2010), generalized Gumbel (GG) (Cooray 2010) and EEL (Ghosh and Alzaatreh 2018). Table 1 summarizes the ranges of skewness and kurtosis of these distributions. It is shown that the E-L $\{G W\}$ fits the widest range of skewness and kurtosis with the exception that the PRHL can fit platykurtic distributions.

\section{Estimation and simulation}

\section{Estimation}

In this subsection, we discuss the maximum likelihood estimation method for the parameters of E-L $\{\mathrm{GW}\}$ distribution. Let $x_{1}, x_{2}, \ldots, x_{n}$ be a random sample from E-L $\{\mathrm{GW}\}$ distribution with parameters $\boldsymbol{\theta}=(\xi, \mu, \sigma)^{t}$, the log-likelihood function is given by

$$
\begin{aligned}
\ell(\boldsymbol{\theta}, \boldsymbol{x})=-n \log |\sigma| & +\sum_{i=1}^{n}\left(\frac{x_{i}-\mu}{\sigma}\right)+(\xi-1) \sum_{i=1}^{n} \log \left[1+\exp \left(\frac{x_{i}-\mu}{\sigma}\right)\right] \\
& +\frac{n}{\xi}-\frac{1}{\xi} \sum_{i=1}^{n}\left[1+\exp \left(\frac{x_{i}-\mu}{\sigma}\right)\right]^{\xi} .
\end{aligned}
$$

Letting $z_{i}=\exp \left(\left(x_{i}-\mu\right) / \sigma\right)$, the score function of the distribution parameters is given by $U_{n}(\boldsymbol{\theta})=(\partial \ell / \partial \xi, \partial \ell / \partial \mu, \partial \ell / \partial \sigma)$, where

$$
\begin{aligned}
& \frac{\partial \ell}{\partial \xi}=-\frac{1}{\xi} \sum_{i=1}^{n}\left[\left(z_{i}+1\right)^{\xi} \log \left(z_{i}+1\right)\right]+\frac{1}{\xi^{2}} \sum_{i=1}^{n}\left[z_{i}+1\right]^{\xi}-\frac{n}{\xi^{2}}+\sum_{i=1}^{n} \log \left(z_{i}+1\right), \\
& \frac{\partial \ell}{\partial \mu}=\frac{1}{\sigma} \sum_{i=1}^{n}\left[z_{i}\left(z_{i}+1\right)^{\xi-1}\right]-\frac{1}{\sigma} \sum_{i=1}^{n} \frac{\xi z_{i}+1}{z_{i}+1} \\
& \frac{\partial \ell}{\partial \sigma}=\frac{1}{\sigma} \sum_{i=1}^{n}\left[z_{i} \log z_{i}\left(z_{i}+1\right)^{\xi-1}\right]-\sum_{i=1}^{n} \frac{\left(1+\xi \log z_{i}\right) z_{i}+1+\log z_{i}}{\sigma\left(z_{i}+1\right)} .
\end{aligned}
$$

The maximum likelihood estimates (MLEs) of the parameters can be obtained by solving the nonlinear Eqs. (16), (17) and (18). The initial values of $\mu$ and $\sigma$ are taken to be the mean and \pm standard deviation of the data respectively. The initial value of $\sigma$ is taken as $s$ (or $-s$ ) if the data is skewed left (or right). The initial value of $\xi$ is taken to be 1.

\section{Simulation}

A simulation study is conducted to explore the performance of the MLE for the parameters of the E-L $\{G W\}$ distribution. Many combinations of the parameters of the E-L $\{G W\}$ model, namely, highly, moderately, and weakly left (or right) skewed, are considered and represent all possible shapes of the model. Different sample sizes $n=\{50,100$, $200,500,1000\}$ are also considered. The MLE of the parameters $\xi, \mu$ and $\sigma$ are computed for 200 repetitions in order to calculate the bias and the standard deviation (SD) for each set of parameter combinations and sample size. Table 2 shows the results of the simulation, and Figs. 5 and 6 present the illustrations. The results show that the bias and SD decrease as the sample size increases. The estimated PDF curve also moves 
Table 2 Bias and SD of the parameter estimates using MLE method

\begin{tabular}{|c|c|c|c|c|c|c|c|c|c|}
\hline \multicolumn{3}{|c|}{ Parameters } & \multirow{2}{*}{$\begin{array}{l}\text { Sample } \\
\text { size }\end{array}$} & \multicolumn{3}{|l|}{ Bias } & \multicolumn{3}{|l|}{$\underline{S D}$} \\
\hline$\xi$ & $\mu$ & $\sigma$ & & $\hat{\xi}-\xi$ & $\hat{\mu}-\mu$ & $\hat{\sigma}-\sigma$ & $S D(\hat{\xi})$ & $S D(\hat{\mu})$ & $S D(\hat{\sigma})$ \\
\hline \multirow[t]{5}{*}{0.5} & \multirow[t]{5}{*}{-1} & \multirow[t]{5}{*}{-1} & 50 & 0.2585 & -0.1125 & -0.0425 & 0.5947 & 0.3552 & 0.2162 \\
\hline & & & 100 & 0.0979 & -0.0386 & -0.0160 & 0.3570 & 0.2413 & 0.1550 \\
\hline & & & 200 & 0.0654 & -0.0278 & -0.0142 & 0.2278 & 0.1587 & 0.1187 \\
\hline & & & 500 & 0.0265 & -0.0070 & -0.0050 & 0.1357 & 0.0999 & 0.0687 \\
\hline & & & 1000 & 0.0102 & 0.0003 & -0.0010 & 0.0928 & 0.0662 & 0.0474 \\
\hline \multirow[t]{5}{*}{2} & \multirow[t]{5}{*}{0} & \multirow[t]{5}{*}{-2} & 50 & 1.0074 & -0.4525 & -0.1085 & 2.5277 & 1.3595 & 0.6366 \\
\hline & & & 100 & 0.3699 & -0.1655 & -0.0345 & 1.4639 & 0.8721 & 0.4376 \\
\hline & & & 200 & 0.3072 & -0.1583 & -0.0494 & 1.1224 & 0.6916 & 0.3598 \\
\hline & & & 500 & 0.1615 & -0.0767 & -0.0279 & 0.8690 & 0.5048 & 0.2375 \\
\hline & & & 1000 & 0.0439 & -0.0156 & -0.0061 & 0.4078 & 0.2709 & 0.1442 \\
\hline \multirow[t]{5}{*}{6} & \multirow[t]{5}{*}{2} & \multirow[t]{5}{*}{-3} & 50 & -1.3031 & 0.9211 & 0.4806 & 2.9698 & 1.5575 & 0.7229 \\
\hline & & & 100 & -0.8896 & 0.6087 & 0.2896 & 2.6342 & 1.3797 & 0.6079 \\
\hline & & & 200 & -0.4115 & 0.3154 & 0.1566 & 2.2743 & 1.1780 & 0.5040 \\
\hline & & & 500 & -0.4251 & 0.2871 & 0.1219 & 1.8211 & 0.9216 & 0.3595 \\
\hline & & & 1000 & -0.4952 & 0.2921 & 0.1137 & 1.3108 & 0.6636 & 0.2506 \\
\hline \multirow[t]{5}{*}{0.5} & \multirow[t]{5}{*}{-1} & \multirow[t]{5}{*}{1} & 50 & 0.2017 & 0.0813 & 0.0291 & 0.5106 & 0.3185 & 0.2133 \\
\hline & & & 100 & 0.0981 & 0.0387 & 0.0161 & 0.3569 & 0.2412 & 0.1550 \\
\hline & & & 200 & 0.0659 & 0.0283 & 0.0141 & 0.2289 & 0.1591 & 0.1189 \\
\hline & & & 500 & 0.0271 & 0.0074 & 0.0054 & 0.1364 & 0.1004 & 0.0688 \\
\hline & & & 1000 & 0.0116 & 0.0004 & 0.0018 & 0.0920 & 0.0662 & 0.0475 \\
\hline \multirow[t]{5}{*}{2} & \multirow[t]{5}{*}{0} & \multirow[t]{5}{*}{2} & 50 & 0.9262 & 0.4091 & 0.0931 & 2.3871 & 1.2927 & 0.6084 \\
\hline & & & 100 & 0.3652 & 0.1627 & 0.0334 & 1.4761 & 0.8769 & 0.4393 \\
\hline & & & 200 & 0.3072 & 0.1585 & 0.0513 & 1.1224 & 0.6939 & 0.3609 \\
\hline & & & 500 & 0.1562 & 0.0713 & 0.0251 & 0.9162 & 0.5125 & 0.2358 \\
\hline & & & 1000 & 0.0365 & 0.0106 & 0.0034 & 0.4089 & 0.2720 & 0.1444 \\
\hline \multirow[t]{5}{*}{6} & \multirow[t]{5}{*}{4} & \multirow[t]{5}{*}{3} & 50 & 1.0664 & 0.0219 & -0.1725 & 6.0035 & 2.5586 & 0.9517 \\
\hline & & & 100 & 0.8875 & 0.1094 & -0.0699 & 5.0824 & 2.2413 & 0.8278 \\
\hline & & & 200 & 0.6818 & 0.1183 & -0.0310 & 3.9748 & 1.7626 & 0.6446 \\
\hline & & & 500 & 0.4806 & 0.0767 & -0.0105 & 3.7760 & 1.6030 & 0.5314 \\
\hline & & & 1000 & 0.1190 & -0.0384 & -0.0374 & 2.7782 & 1.2040 & 0.3957 \\
\hline
\end{tabular}

closer to the actual curve with the increase in the sample size. These results indicate that the MLE method can be used to estimate the parameters of the E-L \{GW\} distribution.

\section{Generalized logistic regression model based on E-L \{GW\}}

In this section, we propose a generalized logistic regression model by assuming the response $Y$ follows E-L $\{\mathrm{GW}\}$ distribution. If the variable of interest is non-negative such as survival time, $T$, then the response $Y$ is defined as $\log (T)$. In the following, we derive a generalized logistic regression model for modeling life-time data. Univariate survival functions and censored data regression problems can be estimated using parametric models for covariate effects. Parametric models produce precise estimates of the quantities of interest when they provide a good fit to the lifetime data set. The reason is that 


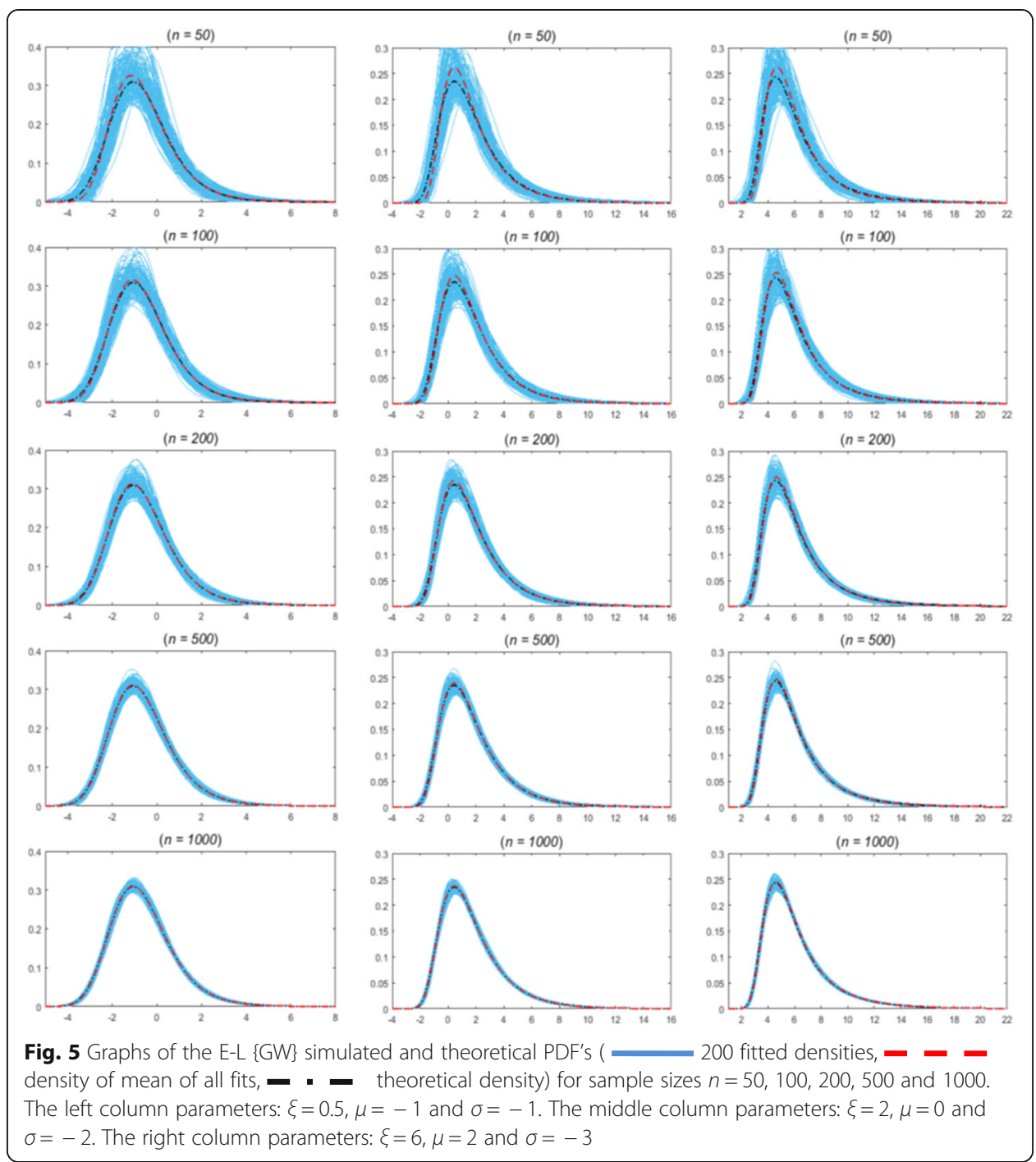

these estimates are based on few parameters in this way. On the basis of the E-L \{GW\} distribution, the following regression model is considered:

$$
y_{i}=\boldsymbol{v}_{i}^{T} \boldsymbol{\beta}+\sigma z_{i}, i=1, \ldots, n,
$$

where the response variable $y_{i}=\log \left(t_{i}\right)$ is the logarithm of the survival time $t_{i}, \boldsymbol{\beta}=\left(\beta_{0}\right.$, $\left.\beta_{1}, \ldots, \beta_{p}\right)^{T}$, and $\sigma \neq 0$ are unknown parameters. Each $y_{i}$ has a covariate vector $\boldsymbol{v}_{i}^{T}=(1$, $\left.v_{i 1}, \ldots, v_{i p}\right)$ that models the linear predictor $\mu_{i}=\boldsymbol{v}_{i}^{T} \boldsymbol{\beta}$. The random error $z_{i}$ has the E-L $\{G W\}$ density (7). The shape parameter $\xi$ can be treated as a nuisance parameter, which may be tested against special cases of the E-L $\{\mathrm{GW}\}$ distribution. It can also be modeled with a vector of covariates $\xi_{i}=\exp \left(\boldsymbol{v}_{i}^{T} \boldsymbol{\gamma}\right)$ that depends on the covariate vector $\boldsymbol{v}_{i}^{T}$ and parameter vector $\gamma=\left(\gamma_{0}, \gamma_{1}, \ldots, \gamma_{p}\right)^{T}$. The corresponding survival function is

$$
S\left(y_{i} \mid \mu(\boldsymbol{v}), \sigma, \xi(\boldsymbol{v})\right)=0.5-\operatorname{sgn}(\sigma)\left(0.5-\exp \left\{\left(1-\left(1+\exp \left(\frac{y_{i}-\boldsymbol{v}_{i}^{T} \boldsymbol{\beta}}{\sigma}\right)\right)^{\exp \left(v_{i}^{T} \gamma\right)}\right) / \exp \left(\boldsymbol{v}_{i}^{T} \boldsymbol{\gamma}\right)\right\}\right) .
$$

The corresponding PDF to the survival function in (20) is given by 


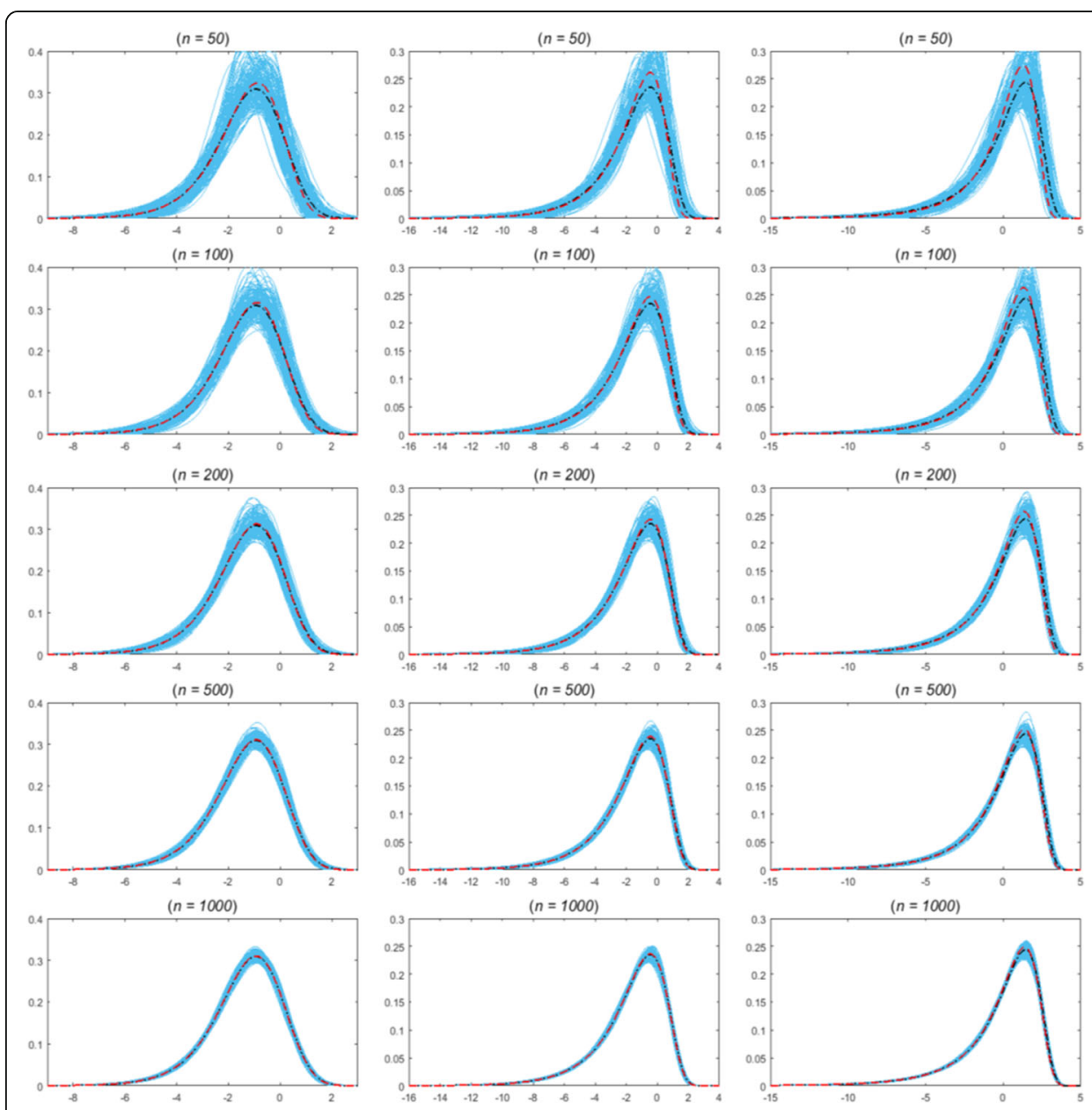

Fig. 6 Graphs of the E-L $\{G W\}$ simulated and theoretical PDF's (

200 fitted densities, $\boldsymbol{-}-\boldsymbol{-}$ density of mean of all fits, - - theoretical density) for sample sizes $n=50,100,200,500$ and 1000 . The left column parameters: $\xi=0.5, \mu=-1$ and $\sigma=1$. The middle column parameters: $\xi=2, \mu=0$ and $\sigma=$ 2. The right column parameters: $\xi=6, \mu=4$ and $\sigma=3$

$$
f\left(y_{i}\right)=\frac{1}{|\sigma|} \exp \left(\frac{y_{i}-\boldsymbol{v}_{i}^{T} \boldsymbol{\beta}}{\sigma}\right)\left(1+\exp \left(\frac{y_{i}-\boldsymbol{v}_{i}^{T} \boldsymbol{\beta}}{\sigma}\right)\right)^{\exp \left(\boldsymbol{v}_{i}^{T} \boldsymbol{\gamma}\right)-1} \exp \left\{\left(1-\left(1+\exp \left(\frac{y_{i}-\boldsymbol{v}_{i}^{T} \boldsymbol{\beta}}{\sigma}\right)\right)^{\exp \left(\boldsymbol{v}_{i}^{T} \boldsymbol{\gamma}\right)}\right) / \exp \left(\boldsymbol{v}_{i}^{T} \boldsymbol{\gamma}\right)\right\}
$$

The generalized logistic regression model consists of many popular regression models as nested models. Some special regression models are as follows:

1. Logistic-log-logistic regression model: this model is obtained as a special case from (20) when $\gamma_{1}=\gamma_{1}=\ldots=\gamma_{p}=0$ and $\gamma_{0} \rightarrow-\infty$ (or $\left.\xi \rightarrow 0\right)$. The survival function is

$$
S(y)=\left\{1+\exp \left(\frac{y-\boldsymbol{v}^{T} \boldsymbol{\beta}}{|\sigma|}\right)\right\}^{-1}
$$

which is the logistic-log-logistic regression model, Lawless (2003, p. 303).

2. Weibull-extreme value regression model: this model is obtained as a special case from (20) when $\gamma_{0}=\gamma_{1}=\ldots=\gamma_{p}=0$ (or $\xi=1$ ), and $\sigma>0$. The survival function is 


$$
S(y)=\exp \left\{-\exp \left(\frac{y-\boldsymbol{v}^{T} \boldsymbol{\beta}}{\sigma}\right)\right\}
$$

which is the classical Weibull regression model, Lawless (2003, p. 296).

3. Log-Fréchet regression model: this model is obtained as a special case from (20) when $\gamma_{0}=\gamma_{1}=\ldots=\gamma_{p}=0$ (or $\xi=1$ ), and $\sigma<0$. The survival function is

$$
S(y)=1-\exp \left\{\exp \left(-\frac{y-\boldsymbol{v}^{T} \boldsymbol{\beta}}{|\sigma|}\right)\right\}
$$

which is the log-Fréchet regression model (Alamoudi et al. 2017).

A sample $\left(y_{1}, v_{1}\right), \ldots,\left(y_{n}, v_{n}\right)$ of $n$ independent observations is considered, where each random response is defined by $y_{i}=\min \left\{\log \left(t_{i}\right), \log \left(c_{i}\right)\right\}$, where $c_{i}$ is the censoring time. We assume non-informative censoring and independent observed lifetimes and censoring times. Let $\Omega$ and $C$ denote the sets of individuals for which $y_{i}$ is the log-lifetime and log-censoring respectively. The total log-likelihood function for the model parameters $\boldsymbol{\theta}=\left(\sigma, \boldsymbol{\beta}^{T}, \boldsymbol{\gamma}^{T}\right)^{T}$ is given as

$$
\ell(\boldsymbol{\theta})=\sum_{i \in \Omega} \log \left[f\left(y_{i}\right)\right]+\sum_{i \in C} \log \left[S\left(y_{i}\right)\right]
$$

where $S\left(y_{i}\right)$ is the survival function in (20) and $f\left(y_{i}\right)$ is the PDF of $S\left(y_{i}\right)$ in (21). The MLE $\hat{\boldsymbol{\theta}}$ of the parameter vector $\boldsymbol{\theta}=\left(\sigma, \boldsymbol{\beta}^{T}, \gamma^{T}\right)^{T}$ of the E-L $\{\mathrm{GW}\}$ regression model can be obtained by maximizing the log-likelihood function in (22).

\section{Applications}

In this section, we apply the E-L $\{\mathrm{GW}\}$ distribution to fit two skewed data and apply the generalized logistic regression to model two censored lifetime data. For the first two data sets, the fits of the E-L $\{G W\}$ distribution are compared with those of other recent generalizations of logistic and Gumbel distributions, namely, the EEL distribution by Ghosh and Alzaatreh (2018), PRHL distribution by Gupta and Kundu (2010), GG by Cooray (2010), and transmuted extreme value (TEV) by Aryal and Tsokos (2009). Maximum likelihood method is used to estimate the model parameters in these applications.

The fitted distributions are compared by using the Akaike information criterion (AIC) and Kolmogorov-Smirnov (KS) statistic and its $p$-value. Data have a good fit when the values of AIC and KS are small, and the $p$-value of KS is large. The plots of the fitted PDFs of some models are demonstrated for visual comparison. Table 3 gives the descriptive statistics of the two data sets. For the third and fourth applications, the generalized logistic regression models are compared with some nested sub-models. The goodness of fits are compared using AIC, the corrected AIC (AICC), and Bayesian

Table 3 The summary statistics of the data sets

\begin{tabular}{llllll}
\hline Data set & No. of Obs. & mean & Std. Dev. & Skewness & Kurtosis \\
\hline Adiponectin & 116 & 10.1809 & 6.8433 & 1.7942 & 6.7108 \\
Turbocharger & 40 & 6.2525 & 1.9555 & -0.6626 & 2.6410 \\
\hline
\end{tabular}


information criterion (BIC) statistics. The estimation process is straightforward, and the $\mathrm{R}$ programming language is used for the first two data sets, while SAS programming language is used for the third and fourth data sets.

\section{Adiponectin data}

The data consist of 116 measurements of Adiponectin from Patrício et al. (2018). The data set is fitted to the E-L $\{G W\}$ model presented in Section 2 and EEL, PRHL, GG, and TEV distributions. Table 4 indicates that the $p$-values of KS statistics of the distributions provide adequate fit to the data. While the five distributions all have three parameters, E-L $\{G W\}$ provides the best fit to the data set. Therefore, the E-L $\{G W\}$ distribution is a better alternate distribution to EEL, PRHL, GG, and TEV distributions. The large skewness and kurtosis of the sample data in Table 3 and the wide range of theoretical skewness and kurtosis in Table 1 suggest that E-L $\{G W\}$ should fit better than other comparable distributions. Figure 7 shows the estimated PDFs of the fitted distributions.

\section{Turbocharger data}

This data set contains the time to failure $\left(10^{3} \mathrm{~h}\right)$ of turbocharger of a type of engine from Xu et al. (2003). These data were studied by Alzaatreh et al. (2016) and Cordeiro et al. (2019) using Weibull-gamma \{log-logistic\} and odd Lomax-Lomax distributions, respectively. For this data set, we fit E-L $\{\mathrm{GW}\}$, EEL, PRHL, GG, and TEV models. The sample data is slightly left-skewed and slightly flatter than normal. It is anticipated that all distributions should fit properly. Table 5 shows all models fit the data set properly, while E-L $\{\mathrm{GW}\}$ has a better fit according to the $p$-values of the KS test statistics. As noticed, the shape parameter estimates of the four distributions that fit better to the data are not statistically significant. This is not surprising since the degree of leftskewness is minor. However, without shape parameter, symmetric distributions do not fit the data properly. Figure 8 shows the fitted models to the turbocharger data set.

\section{Generalized logistic regression model applied to censored class- $\mathrm{H}$ insulation data}

The data are hours to failure of 40 motorettes with a new Class- $\mathrm{H}$ insulation run at $190^{\circ} \mathrm{C}, 220^{\circ} \mathrm{C}, 240^{\circ} \mathrm{C}$, and $260^{\circ} \mathrm{C}$ by Nelson (2004). Midway between the inspection time when the failure is found, and the time of the previous inspection is considered the failure time. The test aims to estimate the median life of such insulation at its design temperature of $180^{\circ} \mathrm{C}$. A median life of over $20,000 \mathrm{~h}$ is desired. The data consist

Table 4 MLEs, their standard errors (SEs) (in parentheses) and goodness of fit measures for the Adiponectin's data set

\begin{tabular}{|c|c|c|c|c|c|c|}
\hline & MLE & & & $-\ell(\hat{\theta})$ & AIC & KS (p-value) \\
\hline $\mathrm{E}-\mathrm{L}\{\mathrm{GW}\}(\mu, \sigma, \zeta)$ & $-3.3614(9.6673)$ & $-8.4609(3.0491)$ & 8.6885 (8.9183) & 354.2757 & 714.5514 & $0.0447(0.9746)$ \\
\hline $\operatorname{EEL}(a, \lambda, \theta)$ & $3.6630(0.6037)$ & $0.2230(0.0215)$ & $1.1250(0.0070)$ & 357.1828 & 720.3655 & $0.0834(0.3950)$ \\
\hline $\operatorname{PRHL}(\mu, \lambda, a)$ & $-12.5762(7.7934)$ & $0.2316(0.0179)$ & $102.6242(177.9828)$ & 360.8723 & 727.7447 & $0.0829(0.4019)$ \\
\hline $\mathrm{GG}(\mu, \sigma, \beta)$ & $7.7414(0.4331)$ & $-2.6167(0.3881)$ & $-0.5155(0.0982)$ & 356.2521 & 718.5042 & $0.0886(0.3224)$ \\
\hline $\operatorname{TEV}(\mu, \sigma, a)$ & $8.3690(0.7213)$ & $4.7540(0.4903)$ & $0.3981(0.2281)$ & 359.6354 & 725.2708 & $0.0717(0.5908)$ \\
\hline
\end{tabular}



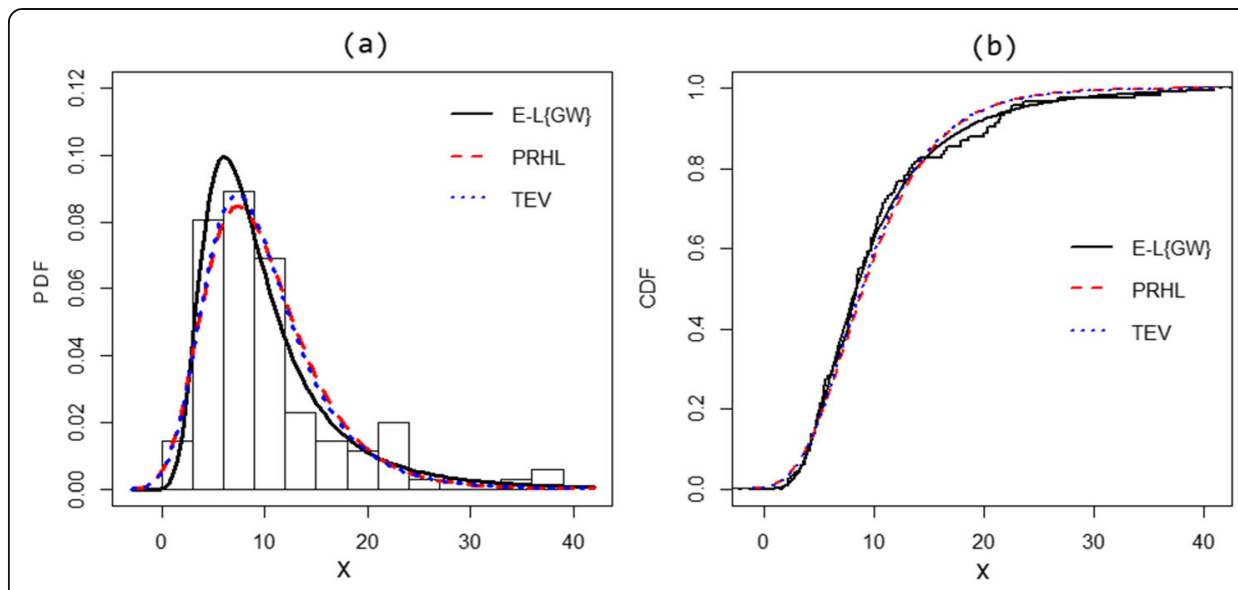

Fig. 7 Adiponectin data set: a Fitted $E-L\{G W\}, G G$ and TEV PDFs. b Fitted $E-L\{G W\}$, GG and TEV CDFs

of $(n=40)$ observations (observed or right censored). The censoring indicator is 0 for censoring and 1 for observed. Each motorette is assigned one of the four test stress levels (10 motorettes in each level). Seven motorettes ( 1 in level 220, 1 in level 240, and 5 in level 260) are lost to follow-up and considered censored. The response variable $y_{i}=$ $\log \left(t_{i}\right)$ is the logarithm of failure times (hours) $t_{i}$ or the logarithm of the censoring time $c_{i}$, and the covariate $v_{i}$ refers to the test stress levels (190, 220, 240, and 260).

The data are analyzed to determine the relationship between $y$ and the level of test stress $(v)$. The following regression model is considered:

$$
y_{i}=\beta_{0}+\beta_{1} v_{i}^{*}+\sigma z_{i}
$$

where $v_{i}^{*}=\left(v_{i}-180\right)$ is the centered stress level obtained by subtracting the design stress value 180, and $y_{i}$ follows the E-L $\{\mathrm{GW}\}$ distribution in (21) with the shape parameter $\xi_{i}=\exp \left(\gamma_{0}+\gamma_{1} v_{i}^{*}\right)$ for $i=1, \ldots, 40$. The model parameters in these applications are estimated by maximum likelihood method. Table 6 indicates that the AIC, AICC, and BIC statistic values of the E-L $\{G W\}$ regression model are smaller than those of the other fitted models. The estimates $\beta_{1}$ and $\gamma_{1}$ are significant at the $5 \%$ level, and the levels of test stress have significant differences. The likelihood ratio (LR) statistic is used to compare the E-L $\{\mathrm{GW}\}$ regression model with some nested models. As shown in Table 6, the E-L $\{\mathrm{GW}\}$ model gives better fit to these data than the other nested models. Table 7 shows the LR statistics and the corresponding $p$-values. The

Table 5 MLEs, their SEs (in parentheses) and goodness of fit measures for the turbocharger data set

\begin{tabular}{|c|c|c|c|c|c|c|}
\hline & MLE & & & $-\ell(\hat{\theta})$ & $\mathrm{AlC}$ & KS ( $p$-value) \\
\hline $\mathrm{E}-\mathrm{L}\{\mathrm{GW}\}(\mu, \sigma, \zeta)$ & $7.7777(1.1696)$ & $1.8229(0.5912)$ & $1.9295(1.8198)$ & 80.0798 & 166.1595 & $\overline{9807)}$ \\
\hline $\operatorname{EEL}(a, \lambda, \theta)$ & 161.0992 (725.8089) & $4.0828(6.0810)$ & $5.8891(6.8837)$ & 87.1234 & 180.2468 & $0.1315(0.4930)$ \\
\hline $\operatorname{PRHL}(\mu, \lambda, a)$ & $8.6753(0.3141)$ & $0.1959(0.1672)$ & $0.0801(0.0769)$ & 80.4757 & 166.9513 & $0.1141(0.6750)$ \\
\hline $\mathrm{GG}(\mu, \sigma, \beta)$ & $-66.6650(299.6159)$ & -199.3733 (817.4937) & -128.7707 (528.6058) & 83.7637 & 173.5274 & 0.0899 (0.9038) \\
\hline $\operatorname{TEV}(\mu, \sigma, a)$ & $4.5182(0.4648)$ & $1.9043(0.2130)$ & $-0.6244(0.2855)$ & 87.8765 & 181.7530 & $0.1421(0.3942)$ \\
\hline
\end{tabular}


(a)

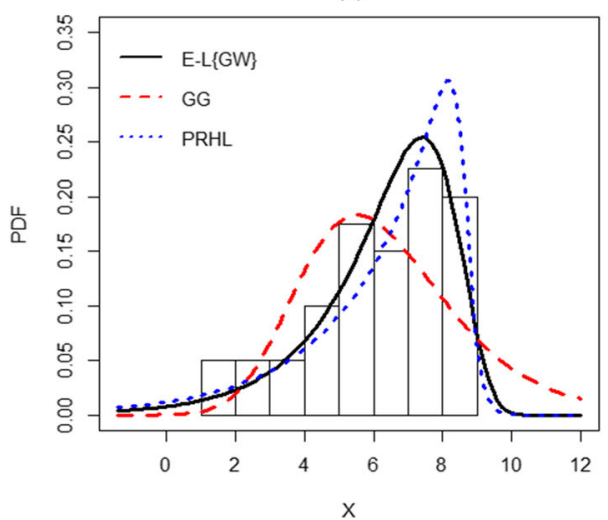

(b)

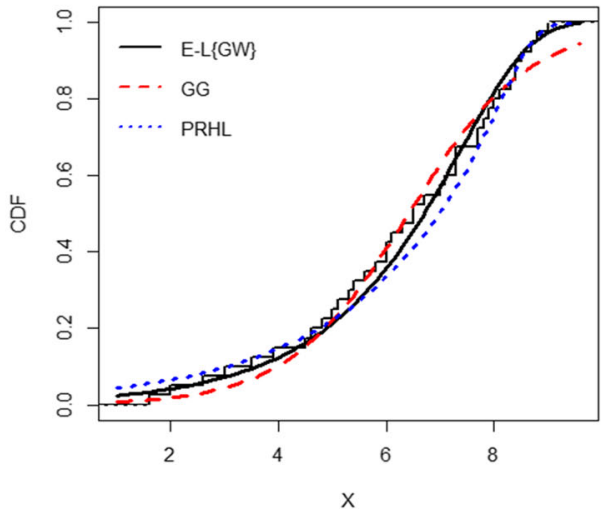

Fig. 8 Turbocharger data set: a Fitted E-L $\{G W\}, G G$ and PRHL PDFs. b Fitted E-L $\{G W\}, G G$ and PRHL CDFs

implication of the results in Table 7 is that the E-L $\{G W\}$ outperforms all the submodels. Thus, one should use the E-L $\{\mathrm{GW}\}$ regression model to analyze the data.

\section{Generalized logistic regression model applied to censored heart transplant data}

The data consist of $n=103$ heart transplant patients of which 69 patients received transplants and 34 did not. The data were from Crowley and $\mathrm{Hu}$ (1977) and reported by Kalbfleisch and Prentice (2002). The data can be used to assess the effect of transplantation on patients' survival. The response variable $y_{i}=\log \left(t_{i}\right)$ is the logarithm of survival time in days (the time from the enrollment until death or until the study ended). The covariates are $v_{i 1}$ (age in years at acceptance) and $v_{i 2}$ (transplant status: $1=$ transplanted, $0=$ not transplanted). The survival status or censoring indicator is 0 for alive and 1 for dead. Thus, the data are analyzed to investigate the relationship between survival time and the covariates age and transplant status. The following regression model is considered:

$$
y_{i}=\beta_{0}+\beta_{1} v_{i 1}+\beta_{2} v_{i 2}+\sigma z_{i}
$$

Table 6 MLEs of the parameters (SE in parentheses), p-values bellow SE, and goodness of fit measures for the Class-H Insulation Data

\begin{tabular}{llllllllll}
\hline Model & $\sigma$ & $\beta_{0}$ & $\beta_{1}$ & $\gamma_{0}$ & $\gamma_{1}$ & $-\ell(\boldsymbol{\theta})$ & AIC & AICC & BIC \\
\hline E-L \{GW\} & -0.2459 & 8.5954 & -0.0165 & 4.6284 & -0.1272 & 5.3258 & 20.6516 & 22.4163 & 29.0960 \\
& $(0.0551)$ & $(0.2845)$ & $(0.0046)$ & $(1.0436)$ & $(0.0299)$ & & & & \\
& & $<.0001$ & 0.0008 & $<.0001$ & $<.0001$ & & & & \\
Logistic & 0.1823 & 9.1328 & -0.02463 & - & - & 14.1221 & 34.2442 & 34.9108 & 39.3108 \\
& $(0.0257)$ & $(0.1024)$ & $(0.0020)$ & & & & & & \\
& & $<.0001$ & $<.0001$ & & & & & & \\
Weibull & 0.2529 & 9.2304 & -0.0239 & - & - & 13.8300 & 33.6600 & 34.3267 & 38.7266 \\
& $(0.0352)$ & $(0.0739)$ & $(0.0014)$ & & & & & & \\
& & $<.0001$ & $<.0001$ & & & & & & \\
Fréchet & -0.3042 & 9.0075 & -0.02501 & - & - & 14.5422 & 35.0845 & 35.7511 & 40.1511 \\
& $(0.0387)$ & $(0.1418)$ & $(0.0028)$ & & & & & & \\
& & $<.0001$ & $<.0001$ & & & & & & \\
\hline
\end{tabular}


Table 7 LR statistics for the Class-H Insulation Data

\begin{tabular}{llll}
\hline Model & Hypotheses & LR statistic & $p$-value \\
\hline E-L $\{G W\}$ vs Logistic & $H_{0}: \gamma_{0}=-\infty, \gamma_{1}=0$ & 17.5925 & 0.0002 \\
E-L $\{G W\}$ vs Weibull & $H_{0}: \gamma_{0}=\gamma_{1}=0, \sigma>0$ & 17.0084 & 0.0002 \\
E-L $\{G W\}$ vs Fréchet & $H_{0}: \gamma_{0}=\gamma_{1}=0, \sigma<0$ & 18.4328 & 0.0001 \\
\hline
\end{tabular}

where $y_{i}$ follows the E-L $\{\mathrm{GW}\}$ distribution in (21) with the shape parameter $\xi_{i}=$ $\exp \left(\gamma_{0}+\gamma_{1} v_{i 1}\right)$ for $i=1, \ldots, 103$. The model parameters in these applications are estimated by maximum likelihood method. Table 8 indicates that the AIC, AICC, and BIC statistic values of the E-L $\{G W\}$ regression model are smaller than those of the other fitted models. The estimates $\beta_{1}, \beta_{2}$, and $\gamma_{1}$ are significant at the $5 \%$ level, and the status of transplant have significant differences. The LR statistic is used to compare the E-L $\{\mathrm{GW}\}$ regression model with some nested models. Table 9 shows the LR statistics and the corresponding $p$-values. As shown in Table 8, the E-L $\{G W\}$ model gives the best goodness of fit statistic among all models.

\section{Summary and conclusions}

The logistic and Gumbel (maximum and minimum) distributions have been widely studied, and many generalizations have been considered to model real-life applications. We propose a new generalization for the logistic and Gumbel distributions called the generalized exponential-logistic distribution. We study the structural properties of this new distribution and the relationships between the parameters and the mean, variance, skewness, and kurtosis. With only three parameters, the E$\mathrm{L}\{\mathrm{GW}\}$ can fit data with a very wide range of skewness (left and right) and kurtosis. The proposed method for developing generalized distributions has a high potential for practitioners. A generalized logistic regression model based on the E$\mathrm{L}\{\mathrm{GW}\}$ distribution is developed. Some existing regression models are sub-models, which makes the generalized logistic regression model a good choice for modeling a wide variety of response variables. Four real data sets are applied to illustrate the usefulness of the new distribution and its regression for fitting skewed data. The applications suggest that these generalized logistic and Gumbel distributions can fit highly skewed data sets effectively.

Table 8 MLEs of the parameters (SEs in parentheses), $p$-values bellow SE and goodness of fit measures for the Heart transplant data set

\begin{tabular}{lllllllllll}
\hline Model & $\sigma$ & $\beta_{0}$ & $\beta_{1}$ & $\beta_{2}$ & $\gamma_{0}$ & $\gamma_{1}$ & $-\ell(\boldsymbol{\theta})$ & AIC & AICC & BIC \\
\hline E-L \{GW\} & -1.4147 & 7.2609 & -0.1050 & 2.9103 & -5.7436 & 0.1108 & 165.5161 & 343.0322 & 343.9072 & 358.8406 \\
& $(0.2824)$ & $(1.3543)$ & $(0.0314)$ & $(0.3407)$ & $(2.6844)$ & $(0.0461)$ & & & & \\
& & $<0.0001$ & 0.0012 & $<0.0001$ & 0.0347 & 0.0180 & & & & \\
Logistic & 1.0231 & 6.0800 & -0.0682 & 2.8609 & - & - & 170.4686 & 348.9372 & 349.3454 & 359.4761 \\
& $(0.0971)$ & $(0.9129)$ & $(0.0197)$ & $(0.3922)$ & & & & & & \\
& & $<0.0001$ & 0.0008 & $<0.0001$ & & & & & & \\
Weibull & 1.4897 & 8.1193 & -0.0951 & 2.8112 & - & - & 173.9400 & 355.8800 & 356.5050 & 364.8164 \\
& $(0.1348)$ & $(0.9845)$ & $(0.0215)$ & $(0.3698)$ & & & & & & \\
& & $<0.0001$ & $<0.0001$ & $<0.0001$ & & & & & & \\
Fréchet & -1.7496 & 4.2179 & -0.0398 & 2.5461 & - & - & 170.6174 & 349.2348 & 349.8598 & 359.7737 \\
& $(0.1501)$ & $(0.9094)$ & $(0.0187)$ & $(0.3695)$ & & & & & & \\
& & $<0.0001$ & 0.0361 & $<0.0001$ & & & & & & \\
\hline
\end{tabular}


Table 9 LR statistics for the Heart transplant data set

\begin{tabular}{llll}
\hline Model & Hypotheses & LR statistic & $p$-value \\
\hline E-L $\{G W\}$ vs Logistic & $H_{0}: \gamma_{0}=-\infty, \gamma_{1}=0$ & 9.9050 & 0.0071 \\
E-L $\{G W\}$ vs Weibull & $H_{0}: \gamma_{0}=\gamma_{1}=0, \sigma>0$ & 16.8478 & 0.0002 \\
E-L $\{G W\}$ vs Fréchet & $H_{0}: \gamma_{0}=\gamma_{1}=0, \sigma<0$ & 10.2026 & 0.0061 \\
\hline
\end{tabular}

\section{Appendix}

\section{Proof of Theorem 1}

The derivative of $f_{X}(x)$ in (7) is given by

$$
f_{X}^{\prime}(x)=-\frac{1}{\sigma|\sigma|}\left(\exp \left(\frac{x-\mu}{\sigma}\right)+1\right)^{\xi-2} \exp \left(\left(\frac{x-\mu}{\sigma}\right)+\left\{1-\left(\exp \left(\frac{x-\mu}{\sigma}\right)+1\right)^{\xi}-1\right\} / \xi\right) w(x)
$$

where $\quad w(x)=\exp \left(\frac{x-\mu}{\sigma}\right)\left(\exp \left(\frac{x-\mu}{\sigma}\right)+1\right)^{\xi}-\xi \exp \left(\frac{x-\mu}{\sigma}\right)-1,-\infty<x<\infty$. By setting $w(x)$ to zero and replacing $\exp \left(\frac{x-\mu}{\sigma}\right)$ by $u$, we obtain (10). If $\xi=\{0,1\}$, then from (10) the mode is at $u=1$, equivalently, $x=\mu$. When $\xi \neq\{0,1\}$, then the curve on the right hand side of (10), $k(u)=u(u+1)^{\xi}$ is convex in $u\left(k^{\prime \prime}(u)>0\right.$ for all $\left.u>0\right)$. Therefore, the curve $k(u)$ and the line $\xi u+1$ on the left hand side of (10) can intersect at most twice. This means $w(x)=0$ has at most two solutions, and so is $\dot{f}(x)=0$. Now, since $\lim _{x \rightarrow-\infty} f_{X}($ $x)=\lim _{x \rightarrow \infty} f_{X}(x)=0$, then $f_{X}(x)$ has exactly one mode. Note that if we assume that $f_{X}(x)$ has two modes (or more), then $w(x)=0$ will have three solutions (two modes and local minimum). This is a contradiction with $w(x)=0$ has at most two solutions, therefore, $f_{X}(x)$ is unimodal.

\section{Proof of Corollary 2}

When $\sigma>0$, the derivative of the hazard function in (9) is given by

$$
h^{\prime}(x)=\frac{1}{\sigma^{2}} \exp \left(\frac{x-\mu}{\sigma}\right)\left\{\xi \exp \left(\frac{x-\mu}{\sigma}\right)+1\right\}\left\{\exp \left(\frac{x-\mu}{\sigma}\right)+1\right\}^{\xi-2} .
$$

From (23), $h^{\prime}(x) \geq 0$ for all $-\infty<x<\infty$, therefore, $h(x)$ is increasing whenever $\sigma>0$. When $\sigma<0$ and by using L'Hopital's rule, we find that

$$
\lim _{x \rightarrow \infty} h(x)=\lim _{x \rightarrow \infty} \frac{\frac{1}{\sigma} \exp \left(\frac{x-\mu}{\sigma}\right)\left\{\exp \left(\frac{x-\mu}{\sigma}\right)+1\right\}^{\xi-1}+(\xi-1) \exp \left(2 \frac{x-\mu}{\sigma}\right)\left\{\exp \left(\frac{x-\mu}{\sigma}\right)+1\right\}^{\xi-2}}{\frac{1}{\sigma}|\sigma| \exp \left(\frac{x-\mu}{\sigma}\right)\left\{\exp \left(\frac{x-\mu}{\sigma}\right)+1\right\}^{\xi-1} \exp \left(\frac{1}{\xi}\left[\left\{\exp \left(\frac{x-\mu}{\sigma}\right)+1\right\}^{\xi}-1\right]\right)}=\frac{1}{|\sigma|} \cdot \square
$$

\section{Proof of Theorem 2}

Let $Z=(X-\mu) / \sigma$, and using binomial expansion, yields

$$
E\left(|X|^{n}\right) \leq \sum_{i=0}^{n}\left(\begin{array}{l}
n \\
i
\end{array}\right)|\mu|^{n-i}|\sigma|^{i} E|Z|^{i}
$$

where $Z$ is $\mathrm{E}-\mathrm{L}\{\mathrm{GW}\}$ random variable with $\mu=0$ and $\sigma=1$.

Now, using definition, we have

$$
E\left(|Z|^{i}\right)=\int_{-\infty}^{\infty}|z|^{i} \exp (z)(1+\exp (z))^{\xi-1} \exp \left\{-\left[(1+\exp (z))^{\xi}-1\right] / \xi\right\} d z
$$




$$
=\int_{-\infty}^{\infty}|\mathrm{z}|^{i} \frac{\exp (\mathrm{z})}{(1+\exp (\mathrm{z}))^{2}} g(\mathrm{z}) d z,
$$

where $g(z)=(1+\exp (z))^{\xi+1} \exp \left\{-\left[(1+\exp (z))^{\xi}-1\right] / \xi\right\}$. By using the elementary calculus, we find that $\sup _{-\infty<z<\infty}\{g(z)\}=e^{-1}(1+\xi)^{1 / \xi+1}$. From (25) we obtain,

$$
E\left(|\mathrm{Z}|^{i}\right) \leq e^{-1}(1+\xi)^{1 / \xi+1} E\left(|L|^{i}\right),
$$

where $E\left(|L|^{i}\right)=\int_{-\infty}^{\infty}|\mathrm{z}|^{i} \frac{\exp (\mathrm{z})}{(1+\exp (\mathrm{z}))^{2}} d z$ is the $i^{\text {th }}$ absolute moment of standard logistic distribution.

Using (26) in (24), the result in (13) is obtained. $\square$

\section{Proof of Theorem 3}

Let $Z=(X-\mu) / \sigma$. We have

$$
E\left(X^{r}\right)=\sum_{n=0}^{r}\left(\begin{array}{l}
r \\
n
\end{array}\right) \mu^{r-n} \sigma^{n} E\left(Z^{n}\right) .
$$

Using Eq. (11), the moments $E\left(Z^{n}\right)$ are obtained as

$$
E\left(Z^{n}\right)=\sum_{i=0}^{\infty} \sum_{j=0}^{\infty} \omega_{i, j} E\left(L_{j+1}^{n}\right)
$$

Therefore, the result in (14) is obtained from (27) directly.

\section{Proof of Proposition 3}

Let $Z=(X-\mu) / \sigma$, then the MGF of $Z$ can be written as

$$
M_{Z}(t)=\int_{-\infty}^{\infty} \exp (z t+z)(1+\exp (\mathrm{z}))^{\xi-1} \exp \left(-\left[(1+\exp (\mathrm{z}))^{\xi}-1\right] / \xi\right) d z .
$$

On setting $u=\left[(1+\exp (z))^{\xi}-1\right] / \xi$ in $(28)$, we obtain

$$
M_{Z}(t)=\int_{0}^{\infty}\left((1+\xi u)^{1 / \xi}-1\right)^{t} \exp (-u) d u .
$$

Using the generalized binomial theorem $(x+y)^{\alpha}=\sum_{i=0}^{\infty} \frac{\Gamma(\alpha+1)}{\Gamma(\alpha-i+1) \Gamma(i+1)} x^{i} y^{\alpha-i},|x|<|y|$,

(29) can be written as

$$
M_{Z}(t)=\sum_{i=0}^{\infty} \frac{\Gamma(t+1)(-1)^{i}}{\Gamma(t-i+1) \Gamma(i+1)} \int_{0}^{\infty}(1+\xi u)^{(t-i) / \xi} \exp (-u) d u .
$$

By using formula (3.382-4) in Gradshteyn and Ryzhik (2000), we obtain

$$
M_{Z}(t)=\sum_{i=0}^{\infty} \frac{\Gamma(t+1)(-1)^{i}}{\Gamma(t-i+1) \Gamma(i+1)} \xi^{(t-i) / \xi} \exp (1 / \xi) \Gamma((t-i) / \xi+1,1 / \xi) .
$$

Now, the MGF of the $X=\mu+\sigma Z$ is defined as

$$
M_{X}(t)=E(\exp (X t))=\exp (\mu t) M_{Z}(\sigma t) .
$$

Using (31) with (30), the result in (15) is obtained. 
Note that the values of the augment $t$ that makes (15) exist can be obtained directly from (29) by noting that $u<\left((1+\xi u)^{1 / \xi}-1\right)<e^{u}$ when $u>0, \quad 0<\xi<1$, and $0<\left((1+\xi u)^{1 / \xi}-1\right)<u$ when $u>0, \xi \geq 1$.

\section{Abbreviations}

AIC: Akaike information criterion; AICC: Corrected AIC; BGL: Beta-generalized logistic; BIC: Bayesian information criterion; CDF: Cumulative distribution function; EEL: Exponentiated-exponential logistic; E-L \{GW\}: Exponential-logistic \{Generalized Weibull\}; ESGN: Extended skew generalized normal; GG: Generalized Gumbel; GN: Generalized normal; HRF: Hazard rate function; KS: Kolmogorov-Smirnov; LR: Likelihood ratio; MGF: Moment generating function; MLEs: Maximum likelihood estimates; PDF: Probability density function; PRHL: Proportional reversed hazard logistic; SD: Standard deviation; SEs: Standard errors; SLD: Skew logistic distribution; SN: Skew normal; TEV: Transmuted extreme value

\section{Acknowledgements}

The authors are very grateful to the handling Editor and the two anonymous reviewers for various constructive comments and suggestions that have greatly improved the presentation of the paper.

\section{Authors' contributions}

The authors, viz. MAA, FF and CL with the consultation of each other carried out this work and drafted the manuscript together. All authors read and approved the final manuscript.

\section{Funding}

There is no funding support for the research work.

\section{Availability of data and materials}

Interested readers can contact the first author.

\section{Competing interests}

On behalf of all authors, the corresponding author states that there is no conflict of interest.

\section{Author details}

'Department of Mathematics, Tafila Technical University, Tafila 66110, Jordan. ${ }^{2}$ Department of Statistics, Actuarial \& Data Sciences, Central Michigan University, Mt. Pleasant, Ml 48859, USA.

Received: 11 March 2020 Accepted: 6 August 2020

Published online: 07 September 2020

\section{References}

Alamoudi, H.H., Mousa, S.A., Baharith, L.A.: Estimation and application in log-Fréchet regression model using censored data. Int. J. Adv. Stat. Probability. 5(1), 23-31 (2017)

Ali, M.M., Pal, M., Woo, J.: Some Exponentiated distributions. Korean Commun. Stat. 14(1), 93-109 (2007)

Aljarrah, M.A., Famoye, F., Lee, C.: A new generalized normal distribution: properties and applications. Commun. Stat. Theory Methods. 48(18), 4474-4491 (2019)

Aljarrah, M.A., Lee, C., Famoye, F.: On generating T-X family of distributions using quantile functions. J. Stat. Distrib. Appl. 1, 2 (2014)

Alzaatreh, A., Lee, C., Famoye, F.: A new method for generating families of continuous distributions. Metron. 71(1), 63-79 (2013)

Alzaatreh, A., Lee, C., Famoye, F.: Family of generalized gamma distributions: properties and applications. Hacettepe J. Math. Stat. 45, 869-886 (2016)

Aryal, R., Tsokos, P.: On the transmuted extreme value distribution with application. Nonlin. Anal. 71(12), 1401-1407 (2009)

Azzalini, A.: A class of distributions which includes the normal ones. Scand. J. Stat. 12, 171-178 (1985)

Balakrishnan, N., Leung, M.Y.: Order statistics from the type I generalized logistic distribution. Commun. Stat. Simul. Comput. $17(1), 25-50(1988)$

Choudhury, K., Abdul, M.M.: Extended skew generalized normal distribution. Metron. 69, 265-278 (2011)

Cooray, K.: Generalized Gumbel distribution. J. Appl. Stat. 37(1), 171-179 (2010)

Cordeiro, G.M., Afify, A.Z., Ortega, E.M.M., Suzuki, A.K., Mead, M.E.: The odd Lomax generator of distributions: properties, estimation and applications. J. Comput. Appl. Math. 347, 222-237 (2019)

Crowley, J., Hu, M.: Covariance analysis of heart transplant data. J. Am. Stat. Assoc. 72, $27-36$ (1977)

Ghosh, l., Alzaatreh, A.: A new class of generalized logistic distribution. Commun. Stat. Theory Methods. 47(9), 2043-2055 (2018)

Gradshteyn, I.S., Ryzhik, I.M.: Table of Integrals, Series, and Products, 6th edn. Academic Press, San Diego (2000)

Gumbel, E.J.: Statistics of Extremes. Columbia University Press, New York (1958)

Gupta, R.D., Kundu, D.: Generalized logistic distributions. J. Appl. Stat. Sci. 18, 51-66 (2010)

Johnson, N.L., Kotz, S., Balakrishnan, N.: Continuous Univariate Distributions: Vol. 1, 2nd edn. John Wiley and Sons, New York (1994)

Johnson, N.L., Kotz, S., Balakrishnan, N.: Continuous Univariate Distributions: Vol. 2, 2nd edn. Wiley, New York (1995)

Kalbfleisch, J.D., Prentice, R.L.: The Statistical Analysis of Failure Time Data, 2nd edn. Wiley, New York (2002)

Lawless, J.F.: Statistical Models and Methods for Lifetime Data, 2nd edn. Wiley, Hoboken New York (2003)

Nadarajah, S.: The skew logistic distribution. Asta Adv. Stat. Anal. 93, 187-203 (2009)

Nassar, M.M., Elmasry, A.: A study of generalized logistic distributions. J. Egypt. Math. Soc. 20(2), 126-133 (2012) 
Nelson, W.B.: Accelerated testing: statistical models, test plans, and data analyses. Wiley, New York (2004)

Patrício, M., Pereira, J., Crisóstomo, J., Matafome, P., Gomes, M., Seiça, R., Caramelo, F.: Using Resistin, glucose, age and BMI to predict the presence of breast cancer. BMC Cancer. 18, 29 (2018). https://doi.org/10.1186/s12885-017-3877-1

Pinheiro, E.C., Ferrari, S.L.: A comparative review of generalizations of the Gumbel extreme value distribution with an application to wind speed data. J. Stat. Comput. Simul. 86(11), 2241-2261 (2016)

Prentice, R.L.: A generalization of the Probit and Logit methods for dose response curves. Biometrics. 32(4), 761-768 (1976)

Stukel, T.: Generalized logistic models. J. Am. Stat. Assoc. 83(402), 426-431 (1988)

Wahed, A.S., Ali, M.M.: The skew-logistic distribution. J. Stat. Res. 35, 71-80 (2001)

Xu, K., Xie, M., Tang, L.C., Ho, S.L.: Application of neural networks in forecasting engine systems reliability. Appl. Soft Comput. 2(4), 255-268 (2003)

\section{Publisher's Note}

Springer Nature remains neutral with regard to jurisdictional claims in published maps and institutional affiliations.

Submit your manuscript to a SpringerOpen ${ }^{0}$ journal and benefit from:

- Convenient online submission

Rigorous peer review

- Open access: articles freely available online

- High visibility within the field

- Retaining the copyright to your article

Submit your next manuscript at $>$ springeropen.com 